\title{
On the Relationships between Input and Output Stability in Linear Two-Ports
}

\author{
Giancarlo Lombardi, Member, IEEE, and Bruno Neri
}

\begin{abstract}
In this paper the relationships between stability at the input and at the output of a linear non-unilateral 2-port are analyzed. For this purpose the existence of a duality mapping between the two ports is shown and, by using the main properties of Möbius transforms, new mutual relationships between the stability conditions at input and output ports are demonstrated. Such relationships add to the case of unconditional stability for which it is well known that unconditional stability at the input implies unconditional stability at the output (and vice versa). This concept will be extended to all the possible cases of reciprocal position between the stability area in the load reflection coefficient plane and the Smith circle, showing that, for a given situation at the output, only one corresponding situation can be observed at the input (and vice versa). Limit cases are further considered.
\end{abstract}

Index Terms - Stability, reflection coefficient, oscillators, $S$-parameters, two-port.

\section{INTRODUCTION}

$\mathrm{T}$ HE problem of the stability of active 2-port circuits represented in terms of $S$-parameters has been thoroughly addressed in the literature, including standard textbooks (see e.g. a former review from the authors in [1]): it represents a crucial issue in the theory of circuits and systems [2],[3]. A classic textbook on microwave circuits [4] performs some treatment of conditional stability, and a more recent one [5] discusses some special cases. Among journal papers, particular attention to the conditional stability issue has been devoted by [6-8]. In particular in [8] criteria based on the wellknown Edwards-Sinsky parameters [9] are listed to assess exhaustively all possible mutual positions of the Smith circle and of the stability circle at the input and output ports, thus indirectly addressing the issue of conditional stability.

The purpose of this paper is to provide simple geometric criteria, which may be used to establish the mutual position of the stability circle and the unit Smith circle at the output

This paragraph of the first footnote will contain the date on which you submitted your paper for review. It will also contain support information, including sponsor and financial support acknowledgment. For example, "This work was supported in part by the U.S. Department of Commerce under Grant BS123456."

G. Lombardi was with the Dipartimento di Ingegneria dell'Informazione (DII), University of Pisa, Pisa I-56122, Italy, and was also with the Scuola Superiore di Studi Universitari e di Perfezionamento "S. Anna", Pisa I-56127, Italy. $\mathrm{He}$ is now with the European Patent Office, Munich, Germany (e-mail: mysgc2004-temporanea@yahoo.it).

B. Neri is with the Radio-Frequency \& Microwave Integrated Circuits Laboratory at the DII, University of Pisa, I-56122 Pisa, Italy (e-mail: bneri@iet.unipi.it). provided that a given mutual position of these circles is observed at the input, and vice versa. These criteria apply just to a non-unilateral 2-port circuit and may be useful in 3-port circuit design and in the project of oscillators. In this latter case, the design could be started by choosing a suitable termination on the third port and deriving that the 2-port circuit, resulting from this choice, is characterized by given convenient mutual positions of the stability and unit Smith circles both at the input and output ports: the choice of the remaining two terminations would be then simplified.

In this paper, after providing notations and definitions related to 2-port circuit stability in Section II, useful properties of the bilinear rational transform or Möbius Transform (MT) in the complex plane are briefly summarized in Section III, whereas in Section IV the existence of a "duality mapping" between the input and the output ports will be shown. Then, in Section V, a topological technique based on this mapping is applied to the case of unconditional instability at a given port and extended to certain significant cases of conditional stability. Finally, the results will be applied in Section VI by sketching the design of a 3-port oscillator and some conclusions are drawn in Section VII.

\section{Definitions RELATED to THE StABILITY OF A 2-PorT}

The following abbreviations and notations will be used with reference to Fig. 1:

$\mathrm{I}=$ Input; $\mathrm{O}=$ Output; $\mathrm{C}=$ Conditional; $\mathrm{U}=$ Unconditional;

$\mathrm{S}=$ Stability; $\mathrm{S}^{*}=$ Instability; $\mathrm{A}=$ Area; $\mathrm{R}=$ Reactive;

$\Gamma=$ Reflection Coefficient; $\Gamma_{S}=$ Source $\Gamma$;

$\Gamma_{L}=\operatorname{Load} \Gamma ; \Gamma_{\text {in }}=\Gamma$ at the Input; $\Gamma_{\text {out }}=\Gamma$ at the Output.

Therefore, the following definitions are made:

IUS: if $\left|\Gamma_{S}\right| \leq 1$ then $\left|\Gamma_{\text {out }}\left(\mid \Gamma_{S}\right)\right|<1$;

OUS: if $\left|\Gamma_{L}\right| \leq 1$ then $\left|\Gamma_{\text {in }}\left(\mid \Gamma_{L}\right)\right|<1$;

US: IUS and OUS;

IUS*: if $\left|\Gamma_{S}\right| \leq 1$ then $\left|\Gamma_{\text {out }}\right| \geq 1$; OUS $*$ :if $\left|\Gamma_{L}\right| \leq 1$ then $\left|\Gamma_{\text {in }}\right| \geq 1$;

ICS: for $\Gamma_{S},\left|\Gamma_{S}\right| \leq 1$, both $\left|\Gamma_{\text {out }}\right|<1$ and $\left|\Gamma_{\text {out }}\right| \geq 1$ may occur;

OCS: for $\Gamma_{L},\left|\Gamma_{L}\right| \leq 1$, both $\left|\Gamma_{i n}\right|<1$ and $\left|\Gamma_{i n}\right| \geq 1$ may occur;

IRUS: if $\left|\Gamma_{S}\right|=1$ then $\left|\Gamma_{\text {out }}\left(\mid \Gamma_{S}\right)\right|<1$, but there exists $\left|\Gamma_{S}\right|<1$ such that $\left|\Gamma_{\text {out }}\right| \geq 1$;

IRUS*: if $\left|\Gamma_{S}\right|=1$ then $\left|\Gamma_{\text {out }}\left(\mid \Gamma_{S}\right)\right| \geq 1$, but there exists $\left|\Gamma_{S}\right|<1$, such that $\left|\Gamma_{\text {out }}\right|<1$

ORUS: if $\left|\Gamma_{L}\right|=1$ then $\left|\Gamma_{\text {in }}\left(\mid \Gamma_{L}\right)\right| 1$, but there exists $\left|\Gamma_{L}\right|<1$, such that $\left|\Gamma_{\text {in }}\right| \geq 1$;

ORUS*: if $\left|\Gamma_{L}\right|=1$ then $\left|\Gamma_{i n}\left(\mid \Gamma_{L}\right)\right| \geq 1$, but there exists $\left|\Gamma_{L}\right|<1$, such that $\left|\Gamma_{\text {out }}\right|<1$; 


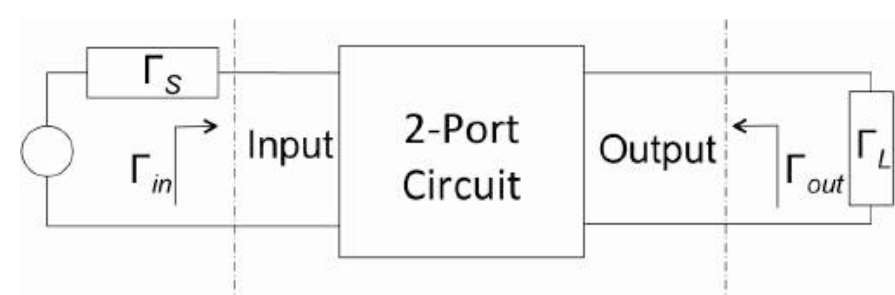

Fig. 1. Notations for a 2-port circuit with reflection coefficients.

ISA: the set of the $\Gamma_{S}$ satisfying $\left|\Gamma_{\text {out }}\left(\Gamma_{S}\right)\right|<1$;

OSA: the set of the $\Gamma_{L}$ satisfying $\left|\Gamma_{i n}\left(\Gamma_{L}\right)\right|<1$;

IS*A: the set of the $\Gamma_{S}$ satisfying $\left|\Gamma_{\text {out }}\left(\Gamma_{S}\right)\right| \geq 1$;

OS*A: the set of the $\Gamma_{L}$ satisfying $\left|\Gamma_{i n}\left(\Gamma_{L}\right)\right| \geq 1$.

The expressions of $\Gamma_{i n}\left(\Gamma_{L}\right)$ and $\Gamma_{\text {out }}\left(\Gamma_{S}\right)$ are recalled here below:

$$
\begin{aligned}
& \Gamma_{\text {in }}=f_{\text {in }}\left(\Gamma_{L}\right)=\frac{S_{11}-\Delta \Gamma_{L}}{1-S_{22} \Gamma_{L}}, \\
& \Gamma_{\text {out }}=f_{\text {out }}\left(\Gamma_{S}\right)=\frac{S_{22}-\Delta \Gamma_{S}}{1-S_{11} \Gamma_{S}},
\end{aligned}
$$

wherein $\mathbf{S}=\left(S_{i j}\right), i, j=1,2$, is the scattering matrix of the S-parameters for the 2-port circuit and $\Delta$ is the determinant of $\mathbf{S}$. It is worth to recall also that $(1 a)$ and $(1 b)$ can be obtained by each other by mutually exchanging both $\Gamma_{S}$ with $\Gamma_{L}$ and 1 with 2 , respectively.

\section{THE MÖBIUS TRANSFORM}

Equations $(1 a)$ and $(1 b)$ involve complex variables and are examples of the well-known bilinear rational transform, also called Möbius transform (MT) [10]. In view of the central role of this transform in the theory of stability of a microwave 2-port circuit, its relevant properties are reviewed here.

The general MT is defined as:

$$
w=\frac{a z+b}{c z+d}=f_{4} \circ f_{3} \circ f_{2} \circ f_{1}(z)
$$

with:

$$
\begin{aligned}
& f_{1}(z)=z+\frac{d}{c} ; f_{2}(z)=\frac{1}{z}=\left(1 / z^{*}\right)^{*} ; \\
& f_{3}(z)=-\frac{D}{c^{2}} z ; f_{4}(z)=z+\frac{a}{c} ; \\
& D=\operatorname{det}\left(\begin{array}{ll}
a & b \\
c & d
\end{array}\right)=a d-b c \neq 0 ;
\end{aligned}
$$

wherein the symbol $f_{l}{ }^{\circ} f_{m}(z)$ denotes the function composition $f_{l}\left[f_{m}(z)\right]$ and each $f_{m}(z), m=1-4$, is clearly a special case of MT. It is clear that ( $1 a)$ is a MT with $w=\Gamma_{i n}, z=\Gamma_{L}, a=-\Delta$, $b=S_{11}, c=-S_{22}, d=1$, and similarly for $(1 b)$.

In the complex plane $f_{1}$ and $f_{4}$ are translations, $f_{2}$ is an inversion in the unit circle $|z|=1$, followed by a reflection across the real axis $\operatorname{Re}(z)=0$ (as defined by the complex conjugation ${ }^{*}$ ) and $f_{3}$ is an homothety (scaling) combined with a rotation around the origin $z=0$. Therefore, in geometrical terms, a MT on the complex plane is a combination of isometries (translations, rotation and reflection, which are preserving shapes and sizes) with a homothety (performing scaling, thus preserving only shape, but not sizes) and an inversion (preserving in general neither shape nor sizes).
From (2) it follows directly that the inverse of a MT is the following MT with the same value of $D$ :

$$
z=\frac{d w-b}{-c w+a},
$$

so that a MT is one-to-one transform, and that the composition of two MTs is a MT.

In view of the properties of the MT summarized up to now, it follows readily [7] that the MT has the following geometric properties, which will be used in the following:

1) It transforms circles into circles (including the special case of a straight line as a circle with its center at infinity, i.e. a straight line may be transformed into a circle or another straight line and vice versa).

2) By being conformal (i.e. the angles between two curves are preserved), it preserves tangency (e.g. between circles and with straight lines) and, if the curves are secant, the number of intersections.

3) It preserves inclusion (if a region is included in another, the same relationship of inclusion applies to the transformed regions).

4) The interior of a curve (e.g. a circle) is fully transformed in the interior or fully transformed in the exterior of the transformed curve depending on the transform coefficients; therefore, to check which of the two possibilities occurs, it is sufficient to check where one single point of the interior is transformed.

\section{ThE DUALITY MAPPING}

From (3) it follows that the transforms inverse to the ones defined in $(2 a)$ and $(2 b)$ are:

$$
\begin{gathered}
\Gamma_{L}=f_{\text {in }}^{-1}\left(\Gamma_{\text {in }}\right)=\frac{\Gamma_{\text {in }}-S_{11}}{S_{22} \Gamma_{\text {in }}-\Delta}, \\
\Gamma_{S}=f_{\text {out }}^{-1}\left(\Gamma_{\text {out }}\right)=\frac{\Gamma_{\text {out }}-S_{22}}{S_{11} \Gamma_{\text {out }}-\Delta},
\end{gathered}
$$

leading to the definition of the Output Stability Circle (4a) and Input Stability Circle (4b), as the images of the unit circles, in the planes $\Gamma_{i n}$ and $\Gamma_{\text {out }}$, in the $\Gamma_{L}$ and $\Gamma_{S}$ planes respectively. This definition, directly deriving from property 1 ) of the MT, is thoroughly discussed in standard textbooks (like [2],[3],[8],[9]).

By rearranging ( $4 a)$ and (4b) and by comparing with the definitions of $f_{\text {in }}$ and $f_{\text {out }}$ in $(1 a)$ and $(1 b)$, one obtains that:

$$
\begin{gathered}
\Gamma_{\text {in }}\left[\frac{1}{\Gamma_{\text {out }}\left(\Gamma_{S}\right)}\right]=\frac{1}{\Gamma_{S}} ; f_{\text {in }}(z)=\frac{1}{f_{\text {out }}^{-1}(1 / z)} ; \\
\Gamma_{\text {out }}\left[\frac{1}{\Gamma_{\text {in }}\left(\Gamma_{L}\right)}\right]=\frac{1}{\Gamma_{L}} ; f_{\text {out }}(z)=\frac{1}{f_{\text {in }}^{-1}(1 / z)} .
\end{gathered}
$$

In other words, in view of $(5 a)$ a pair $\left(\Gamma_{S^{\prime}}, \Gamma_{\text {out }}\right)$ of the input space $\left(\Gamma_{S}, \Gamma_{\text {out }}\right)$ (i.e. such that $\left.\Gamma_{\text {out }}{ }^{\prime}=\Gamma_{\text {out }}\left(\Gamma_{S^{\prime}}\right)\right)$ is mapped to a corresponding pair $\left(1 / \Gamma_{\text {out }}, 1 / \Gamma_{S}{ }^{\prime}\right)$ of the output space $\left(\Gamma_{L}, \Gamma_{\text {in }}\right)$ (i.e. such that $1 / \Gamma_{S^{\prime}}=\Gamma_{\text {in }}\left(1 / \Gamma_{\text {out }}{ }^{\prime}\right)$ ). A corresponding mapping from the output space into the input space, inverse to the previous, is defined in view of $(5 b)$. Equations $(5 a)$ and $(5 b)$ define thus a one-to-one and continuous "duality mapping" between the two spaces $\left(\Gamma_{L}, \Gamma_{\text {in }}\right)$ and $\left(\Gamma_{S}, \Gamma_{\text {out }}\right)$. It is worth observing that the functions appearing in $(5 a)$ and $(5 b)$ are 


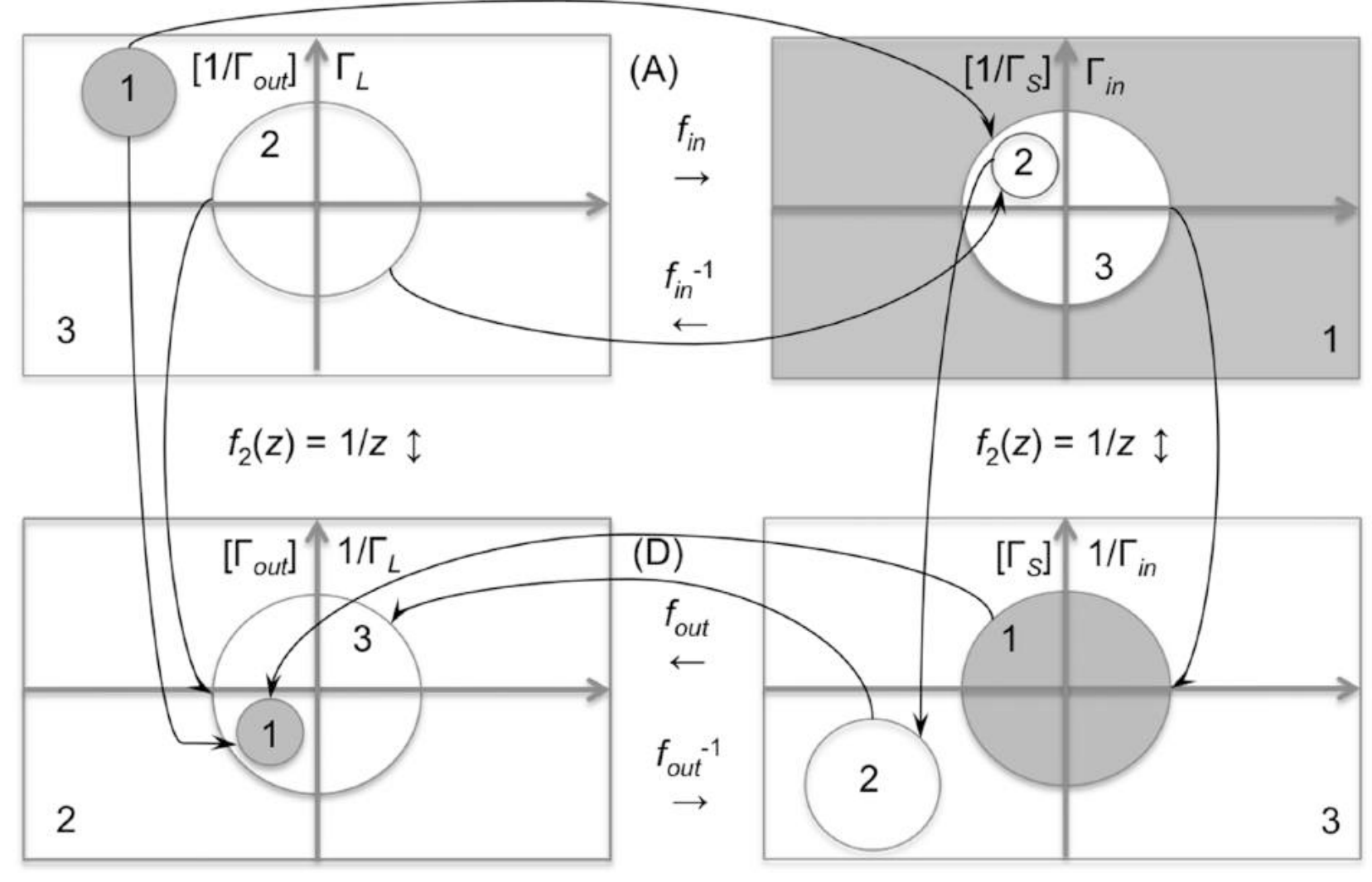

Fig. 2. An example of US: in every plane A to D the disk centered at the origin is the unit disk, i.e. in the plane $\Gamma_{L}$ the region of the passive loads (region 2 in the plane A). The grey region in the plane A, labeled 1 and mapped by $f_{\text {in }}$ in the exterior of the unit disk in the plane $\mathrm{B}$, corresponds by definition to the OS*A, whereas the white region, complementary to the OS*A (union of the regions 2 and 3) and mapped in the unit disk in the plane B, represents the OSA. Planes C and $\mathrm{D}$ are obtained by transforming planes $\mathrm{B}$ and $\mathrm{A}$ with $f_{2}$, respectively. The label on the right of the vertical axis marks then each plane starting from the $\Gamma_{L}$ plane A and mapped from it according to $f_{\text {in }}$ and $f_{2}$. The arrow lines connect each circle with its respective image and the same numeric label $(1,2,3)$ marks corresponding areas in all the transformations. In view of $(5 b)$ one has that the transformation $f_{\text {out }}$ occurs then between planes $\mathrm{C}$ and $\mathrm{D}$ and the $\mathrm{C}$ plane may be identified with the $\Gamma_{S}$ plane (the labels between square brackets on the left of the vertical axes in planes A to D thus apply). Therefore, the region 2 in plane C is mapped by $f_{\text {out }}$ in the exterior of the unit disk in plane $\mathrm{D}$ and corresponds by definition to the IS*A, whereas the union of regions 1 and 3 is mapped inside the unit disk in plane $\mathrm{D}$ and represents the ISA. Starting instead from the $\Gamma_{S}$ plane $\mathrm{C}$ and mapping it first to the other planes $\mathrm{D}, \mathrm{B}, \mathrm{A}$ according to $f_{\text {out }}$ and $f_{2}$, one has similarly that the transformation $f_{\text {in }}$ occurs between the planes A and B in view of $(5 a)$.

MTs, which the properties from 1) to 4) apply to.

A direct consequence of the duality mapping is the wellknown fact that, given a 2-port circuit terminated with the source $\Gamma_{S}{ }^{\prime}$ at the input and the load $\Gamma_{L}{ }^{\prime}$ at the output, one has:

$$
\Gamma_{S}^{\prime} f_{\text {in }}\left(\Gamma_{L}^{\prime}\right)=1 \Leftrightarrow \Gamma_{L}^{\prime} f_{\text {out }}\left(\Gamma_{S}^{\prime}\right)=1
$$

( $\Leftrightarrow$ means "if and only if"). In fact, if $\Gamma_{S}^{\prime} f_{i n}\left(\Gamma_{L}^{\prime}\right)=1$, the pair $\left(\Gamma_{L}^{\prime}, 1 / \Gamma_{S^{\prime}}\right)$ belongs in view of $(1 a)$ to the space $\left(\Gamma_{L}, \Gamma_{i n}\right)$. This pair is mapped by the duality mapping into the pair $\left(\Gamma_{S}^{\prime}, 1 / \Gamma_{L}{ }^{\prime}\right)$ of the space $\left(\Gamma_{S}, \Gamma_{\text {out }}\right)$, which means $\Gamma_{L}^{\prime} f_{\text {out }}\left(\Gamma_{S}^{\prime}\right)=1$ in view of $(1 b)$. The converse implication is shown similarly.

\section{TOPOLOGICAL TREATMENT OF INPUT/OUTPUT STABILITY RELATIONSHIPS}

As an example, the effects of the mapping introduced in the previous section can be observed in Fig. 2, which represents a case of OUS.

In Fig. 2, the output planes $\Gamma_{L}, \Gamma_{i n}, 1 / \Gamma_{i n}, 1 / \Gamma_{L}$ are represented as planes A to D, respectively, and are labeled accordingly to the right of the vertical axis. In every plane, the circle centered at the origin is the unit circle. In the $\Gamma_{L}$ plane A, the grey region (region 1) corresponds to the OS*A, mapped by definition to the exterior of the unit disk in the $\Gamma_{\text {in }}$ plane $\mathrm{B}$, equally labeled as 1 , and region 2 represents the interior of the Smith circle. The remaining points of plane A are region 3 . In the $\Gamma_{L}$ plane $A$, the white region, complementary to region 1 , is the OSA, mapped in the region inside the Smith circle in the $\Gamma_{\text {in }}$ plane B. It is clear that OUS occurs, in that the passive loads are mapped by $f_{\text {in }}$ in the region 2 of plane $\mathrm{B}$ contained in the unit disk. In every transformation of one plane from $\mathrm{A}$ to $\mathrm{D}$ into another plane (in particular, from $\mathrm{A}$ to $\mathrm{B}$ and $\mathrm{D}$, from $\mathrm{B}$ to $\mathrm{C}$ and from $\mathrm{C}$ to $\mathrm{D}$ ), equally numbered or colored regions are transformed into each other (in particular, grey regions are transformed in grey regions and the same applies to white regions). The arrow lines indicate the transformations of significant circles from each plane into another. The application of the MT $f_{2}(z)=1 / z$ to the planes A and B leads to the $1 / \Gamma_{L}$ plane D and to the $1 / \Gamma_{\text {in }}$ plane $\mathrm{C}$, respectively. It is recalled here that $f_{2}$ has the property to be equal to its inverse function, i.e. $f_{2}(z)=1 / z=f_{2}^{-1}(z)$, and that $f_{2}$ transforms the unit circle $|z|=1$ in itself and maps its 


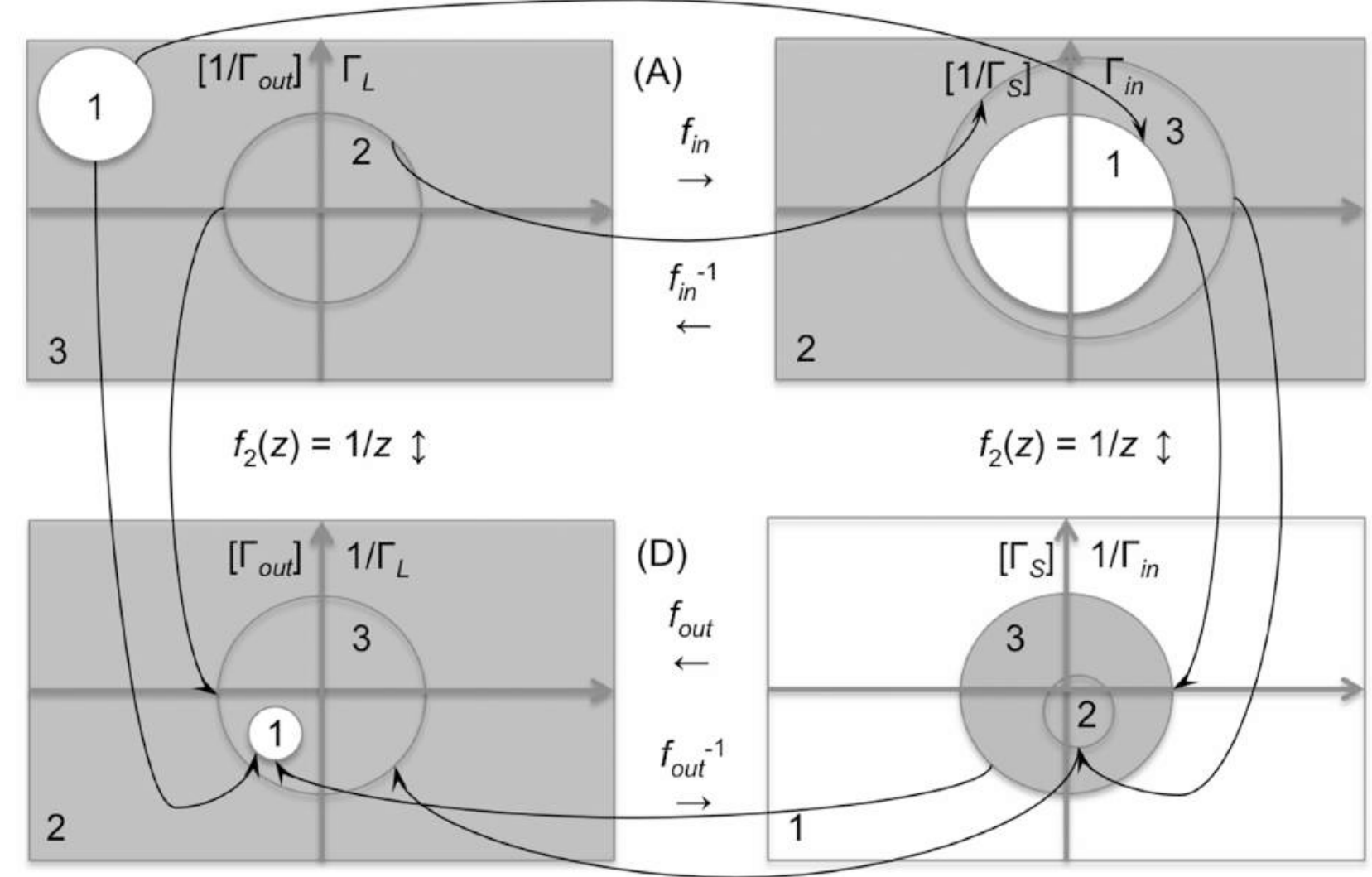

(B)

Fig. 3. An example of OUS* and IRUS: the meaning of the planes A to D and of the white and grey regions is as in Fig. 2. Equally numbered regions are mapped to each other: region 2 corresponds to the IS*A or its image.

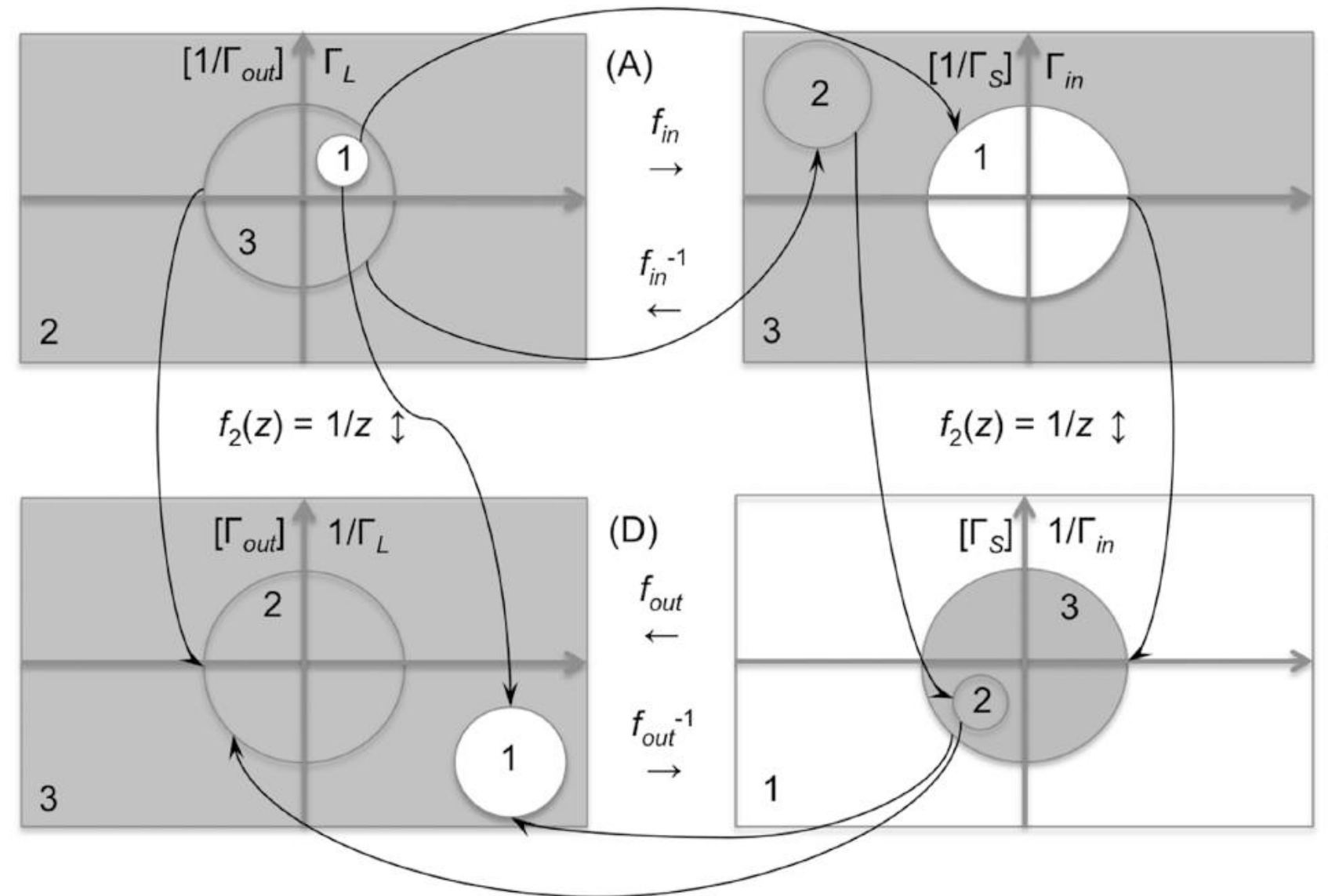

(B)

Fig. 4. An example of reactive unconditional stability (RUI): the meaning of the planes A to D and of the white and grey regions is as in Fig. 2. Equally numbered regions are mapped to each other: region 2 corresponds to the ISA or its image. 
TABLE I

STABILITY AND INSTABILITY CASES

\begin{tabular}{|c|c|}
\hline \hline Input & Output \\
\hline IUS & OUS \\
\hline IUS* & ORUS \\
\hline IRUS & OUS* \\
\hline IRUS* & ORUS* \\
\hline \hline
\end{tabular}

The cases listed in the Table correspond to the Smith circle (or its image) not intersecting the stability circle in any plane. The first line corresponds to US and the last one to RUI. Corresponding limit cases arise when the two circles touch each other (in every plane).

interior in its exterior and vice versa (consider the origin and the point at infinity and use MT property 4$)$ ). It follows that the OS*A, by applying the transformation $f_{\text {in }}$ followed by the transformation $f_{2}$, is mapped in the unit disk of plane $\mathrm{C}$. This is a very general conclusion, which depends directly on the definition of OS*A and applies not only to the situation of US described in Fig. 2. Due to the duality mapping, particularly to $(5 b)$, it follows also in general that the transformation mapping the plane $1 / \Gamma_{\text {in }}$ to the plane $1 / \Gamma_{L}$, i.e. plane $C$ to plane $\mathrm{D}$, is $f_{\text {out }}$, so that the correspondences marked by the arrows between planes $\mathrm{C}$ and $\mathrm{D}$ apply. Therefore, the planes $\mathrm{C}$ and $\mathrm{D}$ may be identified with the $\Gamma_{S}$ and $\Gamma_{\text {out }}$ planes, respectively, and one may conclude immediately that IUS occurs. Indeed, the transformation $f_{\text {out }}$ maps the passive sources (region 1 in the $\Gamma_{S}$ plane C) in region 1 of the $\Gamma_{\text {out }}$ plane $\mathrm{D}$, which results entirely contained inside the unit disk.

As a consequence, the planes A to D (without any alteration of the regions represented therein) may be simply re-labeled as shown in Fig. 2 in square brackets to the left of the vertical axis. In view of the one-to-one character of the duality mapping and of the MTs involved, one could have started equivalently from the input planes $\Gamma_{S}, \Gamma_{\text {out }}, 1 / \Gamma_{\text {out }}, 1 / \Gamma_{S}$ (planes $\mathrm{C}, \mathrm{D}, \mathrm{A}, \mathrm{B})$ obtaining their re-labeling as output planes.

By using Fig. 2 as a reference example, one may conclude with the following general statement, which applies to any case of reciprocal position between stability circle and Smith circle: the transformation with $f_{2}$ of OS*A (IS*A) coincides with the transformation with $f_{\text {out }}\left(f_{\text {in }}\right)$ of the region inside the Smith circle.

Therefore, the application of the properties just formulated to Fig. 2 leads to the conclusion that OUS $\Leftrightarrow$ IUS, the well-known result already shown for instance in [1].

\section{A. Unconditional Instability at a Port}

The well-known case of US discussed in the previous section represents just an example of the potentiality of the topological approach to the analysis of the relationships existing between 2-port input and output for the stability study of the amplifier.

In that respect, let now the case of OUS* be considered: OUS* is equivalent, by definition, to that the region of the passive loads (region 1 in plane A) is properly included in the OS*A. An example of OUS* is presented in Fig. 3, wherein plane A to D have the same meaning as in Fig. 2. Regions corresponding for number or color are again transformed into each other (the OS*A is represented again in grey, whereas the complementary OSA is again in white).

By direct inspection of Fig. 3 one can conclude that IRUS occurs: in fact, the Smith circle of plane $\mathrm{C}\left(\left|\Gamma_{S}\right|=1\right)$ is transformed by $f_{\text {out }}$ in a circle entirely contained inside the Smith circle of plane D $\left(\left|\Gamma_{\text {out }}\right|=1\right)$, whereas some passive source loads are mapped inside (region 3 ) and some other are mapped outside (region 2) the Smith circle in plane D. In a very similar way, by starting from IRUS, OUS* can be obtained so that we can conclude that OUS* and IRUS are equivalent. Similarly, IUS* and ORUS are equivalent as well.

It is worth observing that IUS* is not the condition complementary to IUS, which occurs if at least one $\left|\Gamma_{\mathrm{S}}\right| \leq 1$ does exist for which $\left|\Gamma_{\text {out }}\right| \geq 1$ and is called input potential instability. In case of IUS* for every $\left|\Gamma_{\mathrm{S}}\right| \leq 1$ it must be $\left|\Gamma_{\text {out }}\right| \geq 1$.

\section{B. Reactive Unconditional Instability (RUI)}

Let be finally considered at the particular case of OCS that has been defined in Section II as ORUS*, meaning that for any purely reactive load at the output (i.e. $\left|\Gamma_{L}\right|=1$ ) it is $\left|\Gamma_{i n}\left(\Gamma_{L}\right)\right| \geq 1$, whereas there exist $\left|\Gamma_{L}\right|,\left|\Gamma_{L}\right|<1$, such that $\left|\Gamma_{i n}\left(\Gamma_{L}\right)\right|<1$. An example of this configuration is represented in Fig. 4. In the plane A, seen as output plane $\Gamma_{L}$, ORUS* is by definition equivalent to that the OSA is strictly included in the Smith circle in order to satisfy the definition given in Sec. II. In Fig. 4 the planes $A$ to D have the same meaning as in Fig. 2, wherein regions corresponding for number or color are transformed into each other (the OS*A is represented again in grey, whereas the complementary OSA is again in white).

By direct inspection of Fig. 4 we can conclude that IRUS* occurs: in fact, the Smith circle of plane $\mathrm{C}\left(\left|\Gamma_{S}\right|=1\right)$ is transformed by $f_{\text {out }}$ in a circle entirely exterior to the Smith circle of plane $\mathrm{D}\left(\left|\Gamma_{\text {out }}\right|=1\right)$, so that some passive loads are mapped inside (region 2) and some passive loads are mapped outside (region 3 ) the Smith circle in plane D. In a very similar way, by starting from IRUS*, ORUS* can be obtained, so that it may be concluded that ORUS* and IRUS* are equivalent. In view of that, one may use then the term Reactive Unconditional Instability (RUI) to indicate this situation.

In case of a RUI 2-port circuit, all reactive terminations lead to instability at each port, in the sense that both $\left|\Gamma_{\text {in }}\right|$ and $\left|\Gamma_{\text {out }}\right|$ are not smaller than unity, and this case allows flexible oscillator design.

\section{Further Cases of Conditional Stability}

The considerations made up to now refer to all possible cases arising when the Smith unit circle and the stability circle in one of the planes $\Gamma_{L}(\mathrm{~A})$ or $\Gamma_{S}(\mathrm{C})$ do not intersect with each other and are summarized in Table I. Indeed, in the reasoning in the previous sections it is apparent that, if the two circles do not intersect in one of the planes A to D, the MT character of $f_{2}, f_{\text {in }}$ and $f_{\text {out }}$ and of their inverse functions ensures in view of MT properties 2) and 3) that the images of those circles do not intersect also in the remaining planes. On the other hand, it is 


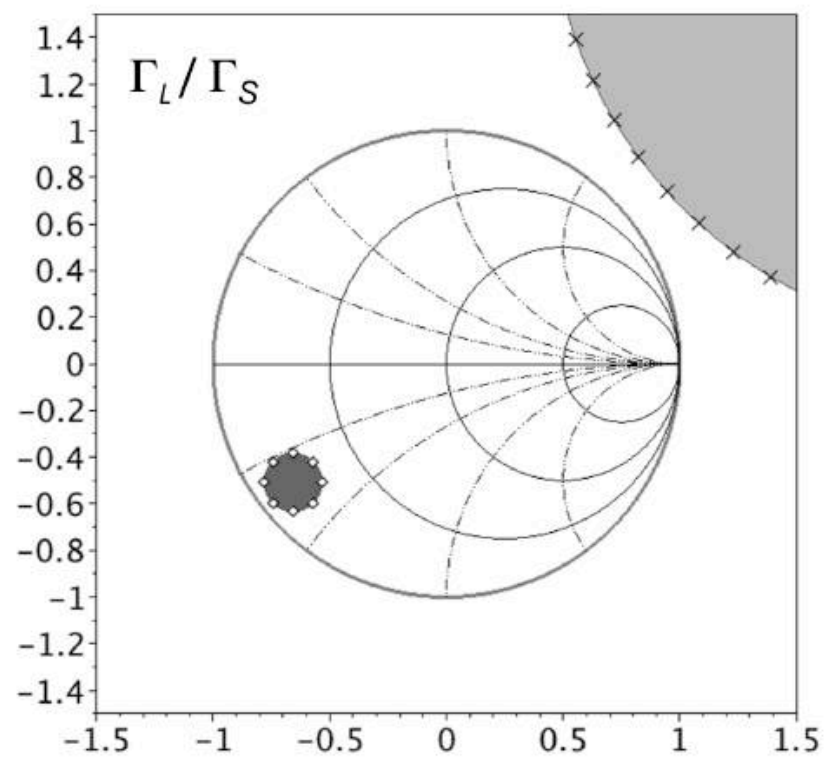

Fig. 5. $\Gamma_{\mathrm{L}}$ and $\Gamma_{\mathrm{S}}$ plane are represented together to show an example of IRUS and OUS*: for the 2-port circuit with matrix $\mathbf{S}^{\prime}$, the OSA in $\Gamma_{\mathrm{L}}$ plane is the grey disk with crosses, while the dark grey disk with diamonds represents the IS*A in $\Gamma_{\mathrm{S}}$ plane.

similarly shown in view of MT property 2) that in the complementary case, when the two circles are secant in a plane, for instance in the $\Gamma_{L}$ plane A, they are secant also in any other plane $A$ to $D$ : such a case always corresponds to conditional stability.

Limit cases of the conditions shown in Table I may be defined, when the two circles touch each other in one of the planes $\Gamma_{L}(\mathrm{~A})$ or $\Gamma_{S}(\mathrm{C})$. In view of property 2 ) of the MT and for corresponding reasons as provided in the non-intersection case, if the Smith unit circle and the stability circle (or their images) are tangent in one of the planes A to D, they will be tangent in any other plane. Therefore, a limit case occurs always at both ports of a 2-port circuit and according to Table I.

\section{ApPLiCATION TO OSCILLATORS}

The conditions of OUS* and IRUS or that of RUI discussed above are useful in the simplification of the project of 2-port oscillators. Indeed, given a suitable 3-port circuit, a 2-port circuit satisfying one of the two conditions may be obtained by opportune termination of the third port. In such a case it is sufficient to put any couple of reactive terminations at the remaining ports, in order to obtain that Barkhausen conditions [11],[12] for the start of oscillation are satisfied.

An example of OUS* 2-port circuit may be derived by using the potentially unstable bipolar transistor of Example 12.2 in [4], having the following common emitter 2-port Sparameters at $6 \mathrm{GHz}$ :

$$
\mathbf{S}=\left[\begin{array}{cc}
0.65 \angle 130^{\circ} & 0.2 \angle 80^{\circ} \\
2 \angle 42^{\circ} & 0.4 \angle-60^{\circ}
\end{array}\right]
$$

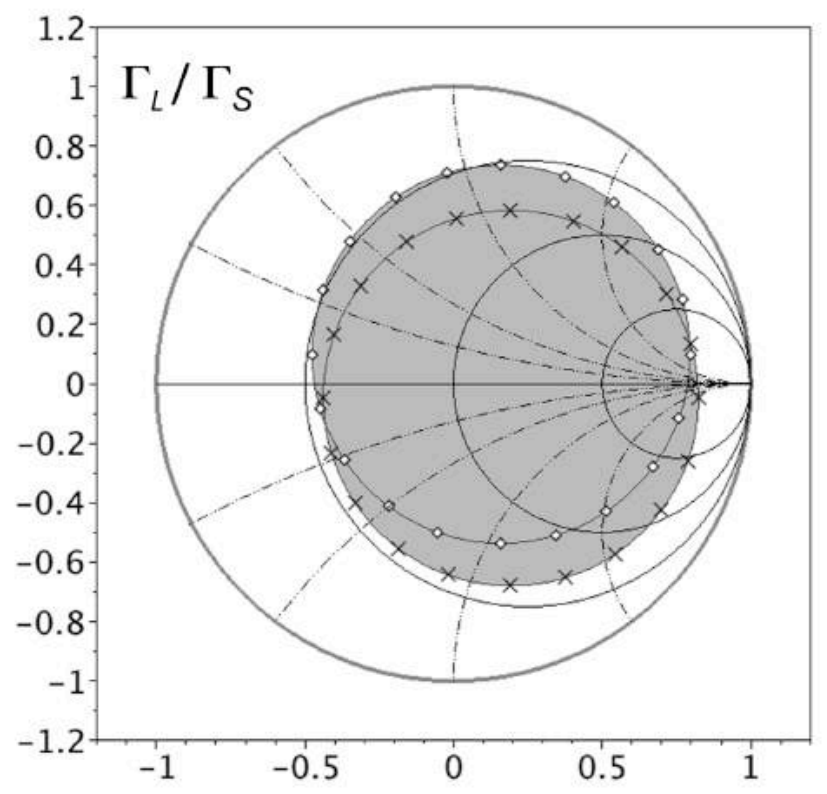

Fig. 6. An example of RUI: for the 2-port circuit with matrix $\mathbf{S}_{2}{ }^{\prime}$, the grey disk labeled with crosses encloses the OSA, while the grey disk labeled with diamonds encloses the ISA.

In fact, by putting at the emitter a capacitive load with $\Gamma_{E}=e^{-j 0.8 \pi}$, the 2-port S-matrix is modified as follows:

$$
\mathbf{S}^{\prime}=\left[\begin{array}{cc}
1.23 \angle 144.8^{\circ} & 0.07 \angle 272.8^{\circ} \\
2.47 \angle 45.9^{\circ} & 0.29 \angle-90.2^{\circ}
\end{array}\right],
$$

with the respective ISA and OSA represented in Fig. 5, showing that the IRUS and OUS* conditions are met. Letting at the collector (output) any passive or purely reactive load, whichever it is, and at the base (input) a purely reactive source $\left(\left|\Gamma_{S}\right|=1\right)$, one has that $\left|\Gamma_{S} \Gamma_{i n}\right|=\left|\Gamma_{i n}\right| \geq 1$, which allows for the start of oscillation at the input.

Let now the following 3-port circuit, studied in [13], be considered, having the following S-parameters:

$$
S_{2}=\left[\begin{array}{ccc}
0.904 \angle-28.51^{\circ} & 0.331 \angle 62.82^{\circ} & 0.148 \angle 68.17^{\circ} \\
1.283 \angle 145.81^{\circ} & 0.592 \angle-25.26^{\circ} & 1.596 \angle-17.06^{\circ} \\
1.3 \angle-12.87^{\circ} & 0.317 \angle-7.55^{\circ} & 0.669 \angle 150.32^{\circ}
\end{array}\right] .
$$

By putting at the third port an inductive load with $\Gamma_{3}=e^{j 0.75 \pi}$ , one obtains the 2-port circuit with the following S-matrix:

$$
\mathbf{S}_{2}{ }^{\prime}=\left[\begin{array}{cc}
0.72 \angle-28.72^{\circ} & 0.33 \angle 70.6^{\circ} \\
2.56 \angle 96.39^{\circ} & 0.71 \angle-16.96^{\circ}
\end{array}\right],
$$

with the respective ISA and OSA represented in Fig. 6, showing that the RUI condition is met. By letting at the input or at the output port a reactive termination, one has $\left|\Gamma_{\text {out }}\right| \geq 1$ or $\left|\Gamma_{\text {in }}\right| \geq 1$, respectively. Moreover, by letting reactive terminations at both input and output port, oscillation starts whichever the values of the reactive terminations are.

\section{CONClusion And Future DeVElopments}

A thorough analysis of instability cases, which may arise 
for a 2-port active circuit allowing sustained oscillation, has been performed. After showing the existence of a duality mapping between the input and the output of the 2-port circuit and recalling properties of Möbius transforms, all the possible cases of reciprocal position between the stability area and Smith circle at the output have been examined in order to obtain the related reciprocal position at the input.

First, in addition to the well-known unconditional stability case, unconditional instability and two special cases of conditional stability (reactive unconditional stability and reactive unconditional instability) have been defined. In this way the relationships between the two ports indicated in Table I have been proved. In particular, it has been shown that a 2-port circuit cannot be made unconditionally instable at both ports, whereas reactive unconditional instability occurs at both ports in analogy with unconditional stability.

Finally, it has been shown, with numerical examples, how potential instability conditions defined and studied here may allow flexible design of oscillator circuits.

However, whereas the criteria for unconditional stability are well consolidated [1-5],[9],[11],[12], further work, now in progress, is required first to provide for analytical criteria involving the S-parameter matrix capable to indicate the occurrence of a given condition among the ones described in Table I. Furthermore, a theoretical tool, now in development, will provide the rules to terminate one of the ports of a given 3 -port active circuit in such a way to obtain a two port with the desired type of conditional stability.

\section{REFERENCES}

[1] G. Lombardi and B. Neri, "Criteria for the evaluation of unconditional stability of microwave linear two-ports: a critical review and new proof", IEEE Trans. on MTT, vol. 47, n. 6, pp. 746-751, Jun. 1999.

[2] D.Woods, "Reappraisal of the Unconditional Stability Criteria for Active 2-Port Networks in Terms of S Parameters", IEEE Trans. Circuits Syst, vol. CAS-23, no. 2, pp. 73-81, Feb. 1976.

[3] R. P. Meys, "Review and Discussion of Stability Criteria for Linear 2-Ports", Trans.Circ.and Syst., vol. 37, no. 11, pp. 1450-1452, Nov. 1990.

[4] R. E. Collin, Foundations for Microwave Engineering, 2nd Edition, Wiley-IEEE Press, 2000, esp. pp. $740 \mathrm{ff}$.

[5] C. Poole, I. Darwazeh, Microwave Active Circuit Analysis and Design, Academic Press, 2016.

[6] D. F. Page and A. R. Boothroyd, "Instability in Two-Port Active Networks", IRE Trans. Circuit Theory, vol. CT-5, no. 2, pp.133-139, June 1958.

[7] R. Kuo and T. Chu, "Instability Analysis of a Three-Port Network", IEEE Trans. on MTT, vol. 59, n.12, pp. 3108-3117, Nov. 2011.

[8] C. Meng, H. Wei, and P. Sun, "Criteria for the evaluation of linear two-port stability using two geometrically derived parameters", Int. J. of Microw. and Wireless Techn., vol. 1, n. 1, pp. 65-72, Feb. 2009.

[9] M. L. Edwards and J. H. Sinsky, "A new criterion for linear 2-port stability using a single geometrically derived parameter", IEEE Trans. on MTT, vol. 40, n. 12, pp. 2303-2311, Dec. 1992.

[10] G. A. Jones and D. Singerman, Complex Functions - An Algebraic and Geometric Viewpoint, Cambridge University Press, 1987.

[11] G. Gonzalez, Microwave Transistor Amplifiers: Analysis and Design, 2nd Edition, Pearson- Prentice Hall, 1997.

[12] R. Mavaddat, Network Scattering Parameters, World Scientific P. C., 1996.

[13] E.L. Tan, "Simplified graphical analysis of linear three-port stability", IEE Proc.-Microw., Ant. and Propag., vol. 152, n. 4, pp. 209-213, Aug. 2005

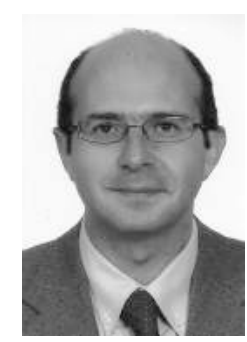

Giancarlo Lombardi was born in Naples, Italy, on December 2, 1971. He received his M.Sc. degree (cum laude) in Telecom Engineering from the University of Pisa, Pisa, Italy, in 1994, and his Ph.D. degree in Information Engineering from the University of Trieste, Trieste, Italy, in 1998.

From 1990 to 1994, he was as a college student at the Sant'Anna School of Advanced Studies (SSSUP), Pisa, Italy. From 1995 to 1998 at Telit, Trieste, Italy, he carried out experimentation and consulting activity about indoor broadband communications. From 1996 to 1999 he cooperated about the modeling and the simulation of the indoor propagation channel with the WINLAB at the Rutgers University, New Brunswick, NJ, USA and with the University of Bologna and the Ugo Bordoni Foundation, Bologna, Italy. In 2000 he was employed at Siemens AG, Bocholt, NRW, Germany, for research in the HIPERLAN candidate standard for WLAN systems. Since the end of 2000 he is patent examiner at the European Patent Office in the domain of video compression and transmission and he has been chief examiner in this domain from 2008 to 2012. His main research interests were in radio channel characterization and modeling techniques for mobile and satellite communications and, starting in 1992 as M.Sc. student, in the theory of microwave circuit stability.

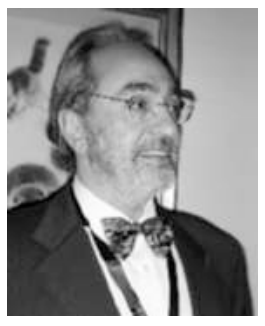

Bruno Neri was born in Reggio Calabria, Italy, in 1956 and received his M.Sc. degree (cum laude) in Electronic Engineering from the University of Pisa in 1980.

In 1983 he joined the Information Engineering Department of this University as a lecturer, in 1992 he became associated professor and since 2000 he is full professor. He teaches courses about Electronic Instrumentation and Measurements, Electronic Devices for Telecommunications, Wireless Electronic Systems, RF and Microwave Integrated Circuit Design. From 2002 to 2010 he has been Director of the Department of Information Engineering (DII) at the University of Pisa. Currently, he is the President of the B.Sc. and M.Sc. Board and Council Member of the Ph.D. Board in Electronic Engineering. Starting from 1999 his research activity has addressed the design of RF integrated circuits and wireless systems for mobile communications, contactless measurements in telemedicine or industrial environments, smart mobility. His current research activity addresses millimeter-wave LNAs, onchip integrated antenna and power amplifiers for Internet of Things scenarios, and low power highly integrated Radar for surveillance and smart mobility applications.

Prof. Neri is co-author of more than 100 papers published in peer-reviewed journals and proceedings and of the book "Highly-Integrated Low-Power Radars", Artech House, Boston, MA, US, 2014. He has been member of the technical expert board in the juridical trial for assessing the dynamics and the responsibilities for the sinking of the cruise ship "Costa Concordia". He received the "Cherubino" Award from the University of Pisa for his scientific and management contributions. 